\title{
MULTILINEAR ISOMETRIES ON FUNCTION ALGEBRAS
}

\author{
MALIHEH HOSSEINI, JUAN J. FONT, AND MANUEL SANCHIS
}

\begin{abstract}
Let $A_{1}, \ldots, A_{k}$ be function algebras (or more generally, dense subspaces of uniformly closed function algebras) on locally compact Hausdorff spaces $X_{1}, \ldots, X_{k}$, respectively, and let $Z$ be a locally compact Hausdorff space. A $k$-linear map $T: A_{1} \times \ldots \times A_{k} \longrightarrow C_{0}(Z)$ is called a multilinear (or k-linear) isometry if

$$
\left\|T\left(f_{1}, \ldots, f_{k}\right)\right\|=\prod_{i=1}^{k}\left\|f_{i}\right\| \quad\left(\left(f_{1}, \ldots, f_{k}\right) \in A_{1} \times \ldots \times A_{k}\right) .
$$

Based on a new version of the additive Bishop's Lemma, we provide a weighted composition characterization of such maps. These results generalize the well-known Holsztyński's theorem ([9]) and the bilinear version of this theorem provided in [10] by a different approach.
\end{abstract}

\section{INTRODUCTION}

Let $X$ be a locally compact Hausdorff space. As usual, $C_{0}(X)$ (resp. $C(X)$ if $X$ is compact) stands for the Banach algebra of all continuous scalar-valued functions on $X$ which vanish at infinity, endowed with the supremum norm, $\|\cdot\|$. In [9], W. Holsztyński inaugurated a new direction of generalization of the famous Banach-Stone Theorem. Namely, he provided the following non-surjective version: If there exists a (not necessarily onto) linear isometry $T: C(X) \longrightarrow C(Y)$, then $T$ is a weighted composition operator on a subset of $Y$. More precisely, there are a closed subset $Y_{0}$ of $Y$, a continuous map $h$ from $Y_{0}$ onto $X$ and a unimodular continuous function $a$ defined on $Y_{0}$ such that $T(f)(y)=a(y) f(h(y))$ for all $y \in Y_{0}$ and all $f \in C(X)$.

In [10], the authors proved, based on the powerful Stone-Weierstrass Theorem, the following bilinear version of Holsztyński's theorem:

Let $T: C(X) \times C(Y) \longrightarrow C(Z)$ be a bilinear (or 2-linear) isometry. Then there exist a closed subset $Z_{0}$ of $Z$, a surjective continuous mapping $\varphi: Z_{0} \longrightarrow X \times Y$ and a unimodular function $a \in C\left(Z_{0}\right)$ such that $T(f, g)(z)=a(z) f\left(\pi_{x}(\varphi(z))\right) g\left(\pi_{y}(\varphi(z))\right)$ for all $z \in Z_{0}$ and every pair $(f, g) \in$ $C(X) \times C(Y)$, where $\pi_{x}$ and $\pi_{y}$ are projection maps.

Key words and phrases: function algebra, $k$-linear isometry, Choquet boundary, additive Bishop's Lemma, peaking function, uniform algebra.

2010 Mathematics Subject Classification. Primary 46J10, 47B38; Secondary 47B33. 
In this paper we extend this bilinear version of Holsztyński's theorem to a more general context, where Stone-Weierstrass Theorem is not applicable. Namely, let $A_{1}, \ldots, A_{k}$ be function algebras (or more generally, dense subspaces of uniformly closed function algebras) on locally compact Hausdorff spaces $X_{1}, \ldots, X_{k}$, respectively, and let $Z$ be a locally compact Hausdorff space. A $k$-linear map $T: A_{1} \times \ldots \times A_{k} \longrightarrow C_{0}(Z)$ is called a multilinear (or k-linear) isometry if

$$
\left\|T\left(f_{1}, \ldots, f_{k}\right)\right\|=\prod_{i=1}^{k}\left\|f_{i}\right\| \quad\left(\left(f_{1}, \ldots, f_{k}\right) \in A_{1} \times \ldots \times A_{k}\right) .
$$

We provide a complete characterization of such maps as follows: given a $k$-linear isometry $T$ : $A_{1} \times \ldots \times A_{k} \longrightarrow C_{0}(Z)$, there exist a nonempty subset $Z_{0}$ of $Z$, a continuous surjective map $\varphi: Z_{0} \longrightarrow C h\left(A_{1}\right) \times \ldots \times C h\left(A_{k}\right)$ and a unimodular continuous function $a: Z_{0} \longrightarrow \mathbb{T}$ such that $T\left(f_{1}, \ldots, f_{k}\right)(z)=a(z) \prod_{i=1}^{k} f_{i}\left(\pi_{i}(\varphi(z))\right)$ for all $\left(f_{1}, \ldots, f_{k}\right) \in A_{1} \times \ldots \times A_{k}$ and $z \in Z_{0}$, where $\pi_{i}$ is the $i$ th projection map.

The main tool we use to prove this characterization is a recent stronger version of the additive Bishop's Lemma (see [12] or Lemma 2.2 below). This technique also lets us fix some inaccuracies detected in [6], particularly in the bounds obtained in the proof of [6, Lemma 3.3]. Furthermore, for the sake of completeness and in order to give a unified version of the proofs involved in this topic, the (known) results for 1-linear isometries are also included and proved straightforwardly by using this version of the additive Bishop's Lemma.

\section{PRELIMINARIES}

Let $X$ be a locally compact Hausdorff space and let $X_{\infty}$ be its one point compactification. Let us recall that $C_{0}(X)$ is the algebra of all continuous scalar-valued functions on $X$ vanishing at infinity. A function algebra $A$ on $X$ is a subalgebra of $C_{0}(X)$ which separates strongly the points of $X$, i.e. for each $x, x^{\prime} \in X$ with $x \neq x^{\prime}$, there exists an $f \in A$ with $f(x) \neq f\left(x^{\prime}\right)$ and for each $x \in X$, there exists an $f \in A$ with $f(x) \neq 0$. If $X$ is a compact Hausdorff space, each unital uniformly closed function algebra on $X$ is called a uniform algebra on $X$.

Let $A$ be a function algebra on a locally compact Hausdorff space $X$. We denote the uniform closure of $A$ by $\bar{A}$. The unique minimal closed subset of $X$ with the property that every function in $A$ assumes its maximum modulus on this set, which exists by [2], is called the $\check{S}$ ilov boundary for $A$ and is denoted by $\partial A$. The Choquet boundary $C h(A)$ of $A$ is the set of all $x \in X$ for which $\delta_{x}$, the evaluation functional at the point $x$, is an extreme point of the unit ball of the dual space of $(A,\|\cdot\|)$. So it is apparent that $C h(A)=C h(\bar{A})$. Besides, note that for a function algebra $A$, $\partial A$ is the closure of $C h(A)$ [2, Theorem 1]. A point $x \in X$ is called a strong boundary point (or weak peak point) for $A$ if for every neighborhood $V$ of $x$, there exists a function $f \in A$ such that $\|f\|=1=|f(x)|$ and $|f|<1$ on $X \backslash V$. It is known that if $A$ is a uniformly closed function algebra 
on a locally compact Hausdorff space $X$, then $C h(A)$ coincides with the set of all strong boundary points (see [11]). However, according to the example given in [4], this coincidence is not true for all function algebras, although the Choquet boundary always contains the strong boundary points.

A function $f \in A$ is a peaking function if $\|f\|=1$ and for each $x \in X$, either $|f(x)|<1$ or $f(x)=1$. If we fix $x_{0} \in X$, then $P_{A}\left(x_{0}\right)$ denotes the set of peaking functions $f$ in $A$ with $f\left(x_{0}\right)=1$. Moreover, if $A$ is a subspace of $C_{0}(X)$, for an element $x \in X$, we set $C_{x}:=\{f \in A:|f(x)|=1=$ $\|f\|\}$. Besides, for $g \in A$ we denote the maximum modulus set of $g$ by $M_{g}:=\{x \in X:|g(x)|=\|g\|\}$.

As mentioned in the introduction, the proofs of the technical lemmas preceding our main result are based essentially on extensions of Bishop's Lemma in the context of uniform algebras [3, Theorem 2.4.1], a result which has been generalized in many directions. Next we include the following generalizations (given in [8] and [12] respectively) which we shall use in the next sections.

Lemma 2.1. Let $A$ be a uniformly closed function algebra on a locally compact Hausdorff space $X$, $f \in A$ and $x_{0} \in C h(A)$. If $f\left(x_{0}\right) \neq 0$, then there exists a peaking function $h \in P_{A}\left(x_{0}\right)$ such that $\frac{f h}{f\left(x_{0}\right)} \in P_{A}\left(x_{0}\right)$.

Proof. The result can be concluded by the arguments similar to [8, Lemma 2.3], where $X$ is a compact Hausdorff space.

Lemma 2.2. Assume that $A$ is a uniformly closed function algebra on a locally compact Hausdorff space $X$ and $f \in A$. Let $x_{0} \in C h(A)$ and arbitrary $r>1$ (or $r \geq 1$ if $f\left(x_{0}\right) \neq 0$ ), then there exists a function $h \in r\|f\| P_{A}\left(x_{0}\right)=\left\{r\|f\| k: k \in P_{A}\left(x_{0}\right)\right\}$ such that

$$
|f(x)|+|h(x)|<\left|f\left(x_{0}\right)\right|+\left|h\left(x_{0}\right)\right|
$$

for every $x \notin M_{h}$ and $|f(x)|+|h(x)|=\left|f\left(x_{0}\right)\right|+\left|h\left(x_{0}\right)\right|$ for all $x \in M_{h}$. Consequently, $\||f|+|h|\|_{X}=$ $\left|f\left(x_{0}\right)\right|+\left|h\left(x_{0}\right)\right|$.

Proof. The proof is exactly the same as that of $[12$, Lemma 1], where $X$ is a compact Hausdorff space.

Let us remark that Lemma 2.1 is a version of the multiplicative Bishop's Lemma and Lemma 2.2 is the strong version of the additive Bishop's Lemma (see [7] for further details concerning Bishop's Lemma).

\section{1-LINEAR ISOMETRIES BETWEEN FUNCTION ALGEBRAS}

In this section we shall assume that $A$ and $B$ are dense subspaces of uniformly closed function algebras on locally compact Hausdorff spaces $X$ and $Y$, respectively, and characterize linear (i.e., 1-linear) isometries $T: A \longrightarrow B$. It should be noted that although these results can be deduced 
from [1], here we provide new shorter proofs based on Lemma 2.2 in order to give a self-contained unified vision of this topic. We refer the reader to [5] for a summary on the study of isometries.

Theorem 3.1. Let $T: A \longrightarrow B$ be a linear isometry. Then there exist a nonempty subset $Y_{0}$ of $Y$, a continuous surjective map $\varphi: Y_{0} \longrightarrow C h(A)$, a unimodular continuous function $a: Y_{0} \longrightarrow \mathbb{T}$, such that $T(f)(y)=a(y) f(\varphi(y))$ for all $f \in A$ and $y \in Y_{0}$. Moreover, $a(y)=T(g)(y)$ for any $g \in A$ with $g(\varphi(y))=1$.

First note that we can extend easily $T: A \longrightarrow B$ to a linear isometry $T: \bar{A} \longrightarrow \bar{B}$ between their uniform closures. Besides, notice that the Choquet boundary for a linear subspace of continuous functions on a locally compact Hausdorff space is defined similar to the function algebra case. So since the Choquet boundary of a subspace equals the Choquet boundary of its uniform closure, without loss of generality, we can assume that $A$ and $B$ are uniformly closed function algebras.

Before providing the proof of Theorem 3.1, we need several lemmas.

Lemma 3.2. Let $x \in C h(A)$. Then the set $\mathcal{I}_{x}:=\bigcap_{f \in C_{x}} M_{T(f)}$ is nonempty.

Proof. The proof is the same as that of [1, Lemma 2.2].

Lemma 3.3. Let $x \in C h(A)$. If $f \in A$ such that $f(x)=0$, then $T(f)(y)=0$ for all $y \in \mathcal{I}_{x}$.

Proof. Let $f \in A$ with $f(x)=0$ and $y \in \mathcal{I}_{x}$. Suppose, on the contrary, that $T(f)(y) \neq 0$. We may assume, without loss of generality, that $\|f\|=1$ and $T(f)(y)=\alpha$, where $0<\alpha \leq 1$. Fix a constant $r>1$. By Lemma 2.2, there is a peaking function $h \in P_{A}(x)$ such that $\||f|+r|h|\|=r$. In particular, $\|f+r \bar{\lambda} h\|=r$, where $\lambda=T(h)(y) \in \mathbb{T}$. Hence

$$
r=\|f+r \bar{\lambda} h\|=\|T(f+r \bar{\lambda} h)\| \geq|T(f)(y)+r|=\alpha+r,
$$

which is a contradiction showing that $T(f)(y)=0$.

Lemma 3.4. If $f \in A$ and $x \in C h(A)$, then $|T(f)(y)|=|f(x)|$ for all $y \in \mathcal{I}_{x}$.

Proof. Let $f \in A, x \in C h(A)$ and $y \in \mathcal{I}_{x}$. If $f(x)=0$, then, by the preceding lemma, $T(f)(y)=0$. Now let us suppose that $f(x) \neq 0$. Since $x \in C h(A)$, there is a peaking function $h \in C_{x}$. If we define

$$
g(t):=f(t)-f(x) h(t) \quad(t \in X)
$$

then $g \in A$ and $g(x)=0$. So, by Lemma 3.3, $0=T(g)(y)=T(f)(y)-f(x) T(h)(y)$. Hence $T(f)(y)=f(x) T(h)(y)$. On the other hand, since $y \in \mathcal{I}_{x}$ and $h \in C_{x},|T(h)(y)|=1$. Therefore, $|T(f)(y)|=|f(x)|$.

Lemma 3.5. For different points $x$ and $x^{\prime}$ in $C h(A), \mathcal{I}_{x} \cap \mathcal{I}_{x^{\prime}}=\emptyset$. 
Proof. Choose a peaking function $f \in C_{x}$ such that $\left|f\left(x^{\prime}\right)\right|<1$. Now if $y \in \mathcal{I}_{x} \cap \mathcal{I}_{x^{\prime}}$, then from Lemma 3.4, it follows that $|T(f)(y)|=|f(x)|=1$ and $|T(f)(y)|=\left|f\left(x^{\prime}\right)\right|<1$, which is a contradiction. Thereby, $\mathcal{I}_{x} \cap \mathcal{I}_{x^{\prime}}=\emptyset$.

Now we are ready to complete the proof of Theorem 3.1:

Proof. Let $Y_{0}:=\bigcup_{x \in C h(A)} \mathcal{I}_{x}$. Clearly, $Y_{0} \neq \emptyset$, by Lemma 3.2. Define the map $\varphi: Y_{0} \longrightarrow C h(A)$ by $\varphi(y):=x$ if $y \in \mathcal{I}_{x}$. Note that, since for different points $x$ and $x^{\prime}$ in $C h(A), \mathcal{I}_{x} \cap \mathcal{I}_{x^{\prime}}=\emptyset$, the map $\varphi$ is well-defined. Furthermore, $\varphi$ is surjective because $\mathcal{I}_{x} \neq \emptyset$ for each $x \in C h(A)$. Meantime, since for all $f \in A,|T(f)|=|f \circ \varphi|$ on $Y_{0}$ and the set $\{|f|: f \in A\}$ separates the points of $X_{\infty}$, it is not difficult to check that $\varphi$ is continuous.

Now we define the function $a: Y_{0} \longrightarrow \mathbb{T}$. For this purpose, let $y \in Y_{0}$. Then take $f \in A$ with $f(\varphi(y))=1$ and define $a(y):=T(f)(y)$. Note that the definition is independent of the choice of $f$ because if $f, f^{\prime} \in A$ and $f(\varphi(y))=1=f^{\prime}(\varphi(y))$, then $f-f^{\prime} \in A$ with $\left(f-f^{\prime}\right)(\varphi(y))=0$. Hence, by Lemma 3.3, we conclude that $T\left(f-f^{\prime}\right)(y)=0$ and so $T(f)(y)=T\left(f^{\prime}\right)(y)$. Moreover, by Lemma 3.4 , it is evident that $|a(y)|=1$.

Next, we give the representation of $T$. Let $f \in A$ and $y \in Y_{0}$. The function $g:=f-f(\varphi(y)) k$, where $k$ is a function in $P_{A}(\varphi(y))$, belongs to $A$ and $g(\varphi(y))=0$. So by Lemma 3.3, $T(f)(y)=$ $f(\varphi(y)) T(k)(y)$, i.e., $T(f)(y)=a(y) f(\varphi(y))$.

We finally show the continuity of $a$. Let $y_{0} \in Y_{0}$ and choose $f \in A$ such that $f\left(\varphi\left(y_{0}\right)\right) \neq 0$. If we define $W:=\{x \in C h(A): f(x) \neq 0\}$, then $\varphi^{-1}(W)$ is a neighborhood of $y_{0}$. Moreover, $a(y)=\frac{T(f)(y)}{(f \circ \varphi)(y)}$ holds for all $y \in \varphi^{-1}(W)$. Now from the continuity of $\frac{T(f)}{f \circ \varphi}$ on $\varphi^{-1}(W)$, it follows that $a$ is also continuous at $y_{0}$.

Remark 3.6. (i) Notice that $\varphi$ sends $C h(T(A))$ onto $C h(A)$. In fact, $T: A \longrightarrow T(A)$ is a bijective isometry, then the adjoint of $T, T^{*}: T(A)^{*} \longrightarrow A^{*}$ is a bijective isometry. Therefore, $\operatorname{ext}\left(T(A)_{1}^{*}\right)$ is sent onto $\operatorname{ext}\left(A_{1}^{*}\right)$, where $T(A)_{1}^{*}$ and $A_{1}^{*}$ are the unit ball of $T(A)^{*}$ and $A^{*}$, respectively. Thus, by Lemma 3.4, it follows easily that $\varphi(C h(T(A))) \subseteq C h(A)$. Next repeating the same arguments for $T^{-1}$ and noting that $\left(T^{-1}\right)^{*}=\left(T^{*}\right)^{-1}$, finally we conclude that $\varphi(C h(T(A)))=C h(A)$. In particular, if $T$ is surjective, then $\varphi$ is a homeomorphism of $C h(B)$ onto $C h(A)$.

(ii) We note that if a map $T: A \longrightarrow C_{0}(Y)$ is defined by $T(f)=$ af $\circ \varphi$ on $Y_{0}$, where $Y_{0} \subseteq Y$ is a boundary for $T(A), a$ is a unimodular continuous function on $Y_{0}$, and $\varphi: Y_{0} \longrightarrow C h(A)$ is a surjective map, then $T$ is a linear isometry. 


\section{4. $k$-LINEAR ISOMETRIES BETWEEN FUNCTION ALGEBRAS}

Let $A_{1}, \ldots, A_{k}$ be dense subspaces of uniformly closed function algebras on locally compact Hausdorff spaces $X_{1}, \ldots, X_{k}$, respectively, and let $Z$ be a locally compact Hausdorff space. We recall that a $k$-linear map $T: A_{1} \times \ldots \times A_{k} \longrightarrow C_{0}(Z)$ is called a multilinear (or $k$-linear) isometry if

$$
\left\|T\left(f_{1}, \ldots, f_{k}\right)\right\|=\prod_{i=1}^{k}\left\|f_{i}\right\| \quad\left(\left(f_{1}, \ldots, f_{k}\right) \in A_{1} \times \ldots \times A_{k}\right) .
$$

In this section we shall deepen in these maps. First note that it is not difficult to extend $T$ : $A_{1} \times \ldots \times A_{k} \longrightarrow C_{0}(Z)$ to a $k$-linear isometry $T: \overline{A_{1}} \times \ldots \times \overline{A_{k}} \longrightarrow C_{0}(Z)$, where $\overline{A_{i}}$ is the uniform closure of $A_{i}(i=1, \ldots, k)$. So, as before, without loss of generality, we can assume each $A_{i}$ $(i=1, \ldots, k)$ is a uniformly closed function algebra.

Let us recall that for an element $x_{i} \in X_{i}$, we set $C_{x_{i}}:=\left\{f \in A_{i}:\left|f\left(x_{i}\right)\right|=1=\|f\|\right\}$. Moreover, for $g \in C_{0}(Z), M_{g}:=\{z \in Z:|g(z)|=\|g\|\}$ stands for the maximum modulus set of $g$.

Lemma 4.1. Let $\left(x_{1}, \ldots, x_{k}\right) \in C h\left(A_{1}\right) \times \ldots \times C h\left(A_{k}\right)$. The set

$$
\mathcal{I}_{x_{1}, \ldots, x_{k}}:=\left\{z \in Z: z \in M_{T\left(f_{1}, \ldots, f_{k}\right)} \text { for all }\left(f_{1}, \ldots, f_{k}\right) \in C_{x_{1}} \times \ldots \times C_{x_{k}}\right\}
$$

is nonempty.

Proof. The proof is a modification of the proof of $\left[6\right.$, Lemma 3.1]. Since for each $\left(f_{1}, \ldots, f_{k}\right) \in$ $C_{x_{1}} \times \ldots \times C_{x_{k}}$, the maximum modulus set of $T\left(f_{1}, \ldots, f_{k}\right), M_{T\left(f_{1}, \ldots, f_{k}\right)}$, is a compact subset of $Z_{\infty}$, so it is enough to check that the family $\left\{M_{T\left(f_{1}, \ldots, f_{k}\right)}:\left(f_{1}, \ldots, f_{k}\right) \in C_{x_{1}} \times \ldots \times C_{x_{k}}\right\}$ has the finite intersection property. For this, let $\left(f_{1}^{1}, \ldots, f_{k}^{1}\right), \ldots,\left(f_{1}^{n}, \ldots, f_{k}^{n}\right)$ be members in $C_{x_{1}} \times \ldots \times C_{x_{k}}$. Define

$$
f_{i}:=\frac{1}{n} \sum_{j=1}^{n} \frac{1}{f_{i}^{j}\left(x_{i}\right)} f_{i}^{j}, \quad i \in\{1, \ldots, k\} .
$$

Clearly, $\left(f_{1}, \ldots, f_{k}\right) \in C_{x_{1}} \times \ldots \times C_{x_{k}}$. Hence $\left\|T\left(f_{1}, \ldots, f_{k}\right)\right\|=\left\|f_{1}\right\| \ldots\left\|f_{k}\right\|=1$. Then there is a point $z_{0} \in Z$ such that

$$
1=\left|T\left(f_{1}, \ldots, f_{k}\right)\left(z_{0}\right)\right|=\frac{1}{n^{k}}\left|\sum_{1 \leq i_{1}, \ldots, i_{k} \leq n} \frac{1}{f_{1}^{i_{1}}\left(x_{1}\right)} \ldots \frac{1}{f_{k}^{i_{k}}\left(x_{k}\right)} T\left(f_{1}^{i_{1}}, \ldots, f_{k}^{i_{k}}\right)\left(z_{0}\right)\right| .
$$

Since for each $1 \leq i_{1}, \ldots, i_{k} \leq n, f_{1}^{i_{1}} \in C_{x_{1}}, \ldots, f_{k}^{i_{k}} \in C_{x_{k}}$ and $\left\|T\left(f_{1}^{i_{1}}, \ldots, f_{k}^{i_{k}}\right)\right\|=1$, we conclude that $\left|T\left(f_{1}^{i_{1}}, \ldots, f_{k}^{i_{k}}\right)\left(z_{0}\right)\right|=1$. In particular, $z_{0} \in \bigcap_{i=1}^{n} M_{T\left(f_{1}^{i}, \ldots, f_{k}^{i}\right)}$. Therefore $\bigcap_{i=1}^{n} M_{T\left(f_{1}^{i}, \ldots, f_{k}^{i}\right)} \neq \emptyset$, as was to be proved.

Lemma 4.2. Fix $i \in\{1, \ldots, k\}$ and let $\left(x_{1}, \ldots, x_{k}\right) \in C h\left(A_{1}\right) \times \ldots \times C h\left(A_{k}\right)$. If $f=\left(f_{1}, \ldots, f_{k}\right) \in$ $C_{x_{1}} \times \ldots \times C_{x_{i-1}} \times A_{i} \times C_{x_{i+1}} \times \ldots \times C_{x_{k}}$ such that $f_{i}\left(x_{i}\right)=0$ and $z \in \mathcal{I}_{x_{1}, \ldots, x_{k}}$ then $T(f)(z)=0$. 
Proof. For simplicity, we can take $i=1$. Let $f=\left(f_{1}, \ldots, f_{k}\right) \in A_{1} \times C_{x_{2}} \times \ldots \times C_{x_{k}}$ such that $f_{1}\left(x_{1}\right)=0$ and suppose that there exists $z_{0} \in \mathcal{I}_{x_{1}, \ldots, x_{k}}$ such that $T(f)\left(z_{0}\right) \neq 0$. We can assume, without loss of generality, that $\left\|f_{1}\right\|=1$ and $T(f)\left(z_{0}\right)=\alpha$, where $0<\alpha \leq 1$. Fix a constant $r>1$. By Lemma 2.2, there is a peaking function $h_{1} \in A_{1}$ such that $h_{1}\left(x_{1}\right)=1$ and $\left\|\left|f_{1}\right|+r\left|h_{1}\right|\right\|=r$. In particular, $\left\|f_{1}+r \bar{\lambda} h_{1}\right\|=r$, where $\lambda=T\left(h_{1}, f_{2}, \ldots, f_{k}\right)\left(z_{0}\right) \in \mathbb{T}$. Then we have

$$
r=\left\|f_{1}+r \bar{\lambda} h_{1}\right\|\left\|f_{2}\right\| \ldots\left\|f_{k}\right\|=\left\|T\left(f_{1}+r \bar{\lambda} h_{1}, f_{2}, \ldots, f_{k}\right)\right\|,
$$

while

$$
T\left(f_{1}+r \bar{\lambda} h_{1}, f_{2}, \ldots, f_{k}\right)\left(z_{0}\right)=T\left(f_{1}, f_{2}, \ldots, f_{k}\right)\left(z_{0}\right)+r \bar{\lambda} T\left(h_{1}, f_{2}, \ldots, f_{k}\right)\left(z_{0}\right)=\alpha+r
$$

a contradiction which yields $T(f)(z)=0$ for all $z \in \mathcal{I}_{x_{1}, \ldots, x_{k}}$.

Lemma 4.3. Let $\left(x_{1}, \ldots, x_{k}\right) \in C h\left(A_{1}\right) \times \ldots \times C h\left(A_{k}\right)$ and $z \in \mathcal{I}_{x_{1}, \ldots, x_{k}}$. Let also $I$ and $J$ be two disjoint sets with $I \neq \emptyset$ and $I \cup J=\{1, \ldots, k\}$. Assume that for each $j \in J, h_{j} \in C_{x_{j}}$ and for each $i \in I, f_{i} \in A_{i}$ with $f_{i}\left(x_{i}\right)=0$, then $T\left(F_{1}, \ldots, F_{k}\right)(z)=0$, where $F_{t}=f_{t}$ if $t \in I$ and $F_{t}=h_{t}$ if $t \in J$.

Proof. Let us suppose, contrary to what we claim, that there exists $z_{0} \in \mathcal{I}_{x_{1}, \ldots, x_{k}}$ such that $T\left(F_{1}, \ldots, F_{k}\right)\left(z_{0}\right) \neq 0$. Without loss of generality, we may assume that $\left\|f_{i}\right\|=1$ for each $i \in I$ and $T\left(F_{1}, \ldots, F_{k}\right)\left(z_{0}\right)=\alpha$ with $0<\alpha \leq 1$. Fix a constant $r>1$. For each $i \in I$, we can choose, by Lemma 2.2, a peaking function $h_{i} \in C_{x_{i}}$ such that $\left\|\left|f_{i}\right|+r\left|h_{i}\right|\right\|=r$. In particular, for each $i \in I$ we have $\left\|f_{i}+r \bar{\lambda} h_{i}\right\|=r$, where $\lambda=T\left(h_{1}, \ldots, h_{k}\right)\left(z_{0}\right) \in \mathbb{T}$.

Let us first suppose that $I=\{1,2\}$. Hence, by Lemma 4.2, we can conclude that

$$
\begin{aligned}
T\left(f_{1}+r \bar{\lambda} h_{1}, f_{2}+r h_{2}, h_{3}, \ldots, h_{k}\right)\left(z_{0}\right) & =T\left(f_{1}, f_{2}, h_{3}, \ldots, h_{k}\right)\left(z_{0}\right)+r \bar{\lambda} T\left(h_{1}, f_{2}, h_{3}, \ldots, h_{k}\right)\left(z_{0}\right) \\
& +r T\left(f_{1}, h_{2}, h_{3}, \ldots, h_{k}\right)\left(z_{0}\right)+r^{2} \bar{\lambda} T\left(h_{1}, \ldots, h_{k}\right)\left(z_{0}\right)=\alpha+r^{2} \\
& >r^{2}=\left\|f_{1}+r \bar{\lambda} h_{1}\right\|\left\|f_{2}+r h_{2}\right\|\left\|h_{3}\right\| \ldots\left\|h_{k}\right\| \\
& =\left\|T\left(f_{1}+r \bar{\lambda} h_{1}, f_{2}+r h_{2}, h_{3}, \ldots . h_{k}\right)\right\|,
\end{aligned}
$$

a contradiction which implies that the result is true when $I=\{1,2\}$. Similarly, this result is held for all the cases where $\operatorname{card}(I)=2$.

Now we can continue by induction: noting to the above explanation, let us assume that the result is true for $\operatorname{card}(I)=l-1$ and $3 \leq l \leq k$. We shall show that the result is held if $\operatorname{card}(I)=l$. We 
suppose that $\operatorname{card}(I)=l$ and $I=\left\{x_{1}, \ldots, x_{l}\right\}$, without loss of generality. If $l<k$, then we get

$$
\begin{aligned}
r^{l} & =\left\|f_{1}+r \bar{\lambda} h_{1}\right\|\left\|f_{2}+r h_{2}\right\| \ldots\left\|f_{l}+r h_{l}\right\|\left\|h_{l+1}\right\| \ldots\left\|h_{k}\right\| \\
& =\left\|T\left(f_{1}+r \bar{\lambda} h_{1}, f_{2}+r h_{2}, \ldots, f_{l}+r h_{l}, h_{l+1}, \ldots, h_{k}\right)\right\| \\
& \geq\left|T\left(f_{1}+r \bar{\lambda} h_{1}, f_{2}+r h_{2}, \ldots, f_{l}+r h_{l}, h_{l+1}, \ldots, h_{k}\right)\left(z_{0}\right)\right| \\
& =\left|T\left(f_{1}, \ldots, f_{l}, h_{l+1}, \ldots, h_{k}\right)\left(z_{0}\right)+r^{l} \bar{\lambda} T\left(h_{1}, \ldots, h_{k}\right)\left(z_{0}\right)\right|=\alpha+r^{l},
\end{aligned}
$$

which is impossible. Therefore, $T\left(f_{1}, \ldots, f_{l}, h_{l+1}, \ldots, h_{k}\right)(z)=0$ for all $z \in \mathcal{I}_{x_{1}, \ldots, x_{k}}$. Now if $l=k$, then $I=\left\{x_{1}, \ldots, x_{k}\right\}$ and

$$
\begin{aligned}
r^{k} & =\left\|f_{1}+r \bar{\lambda} h_{1}\right\|\left\|f_{2}+r h_{2}\right\| \ldots\left\|f_{k}+r h_{k}\right\|=\left\|T\left(f_{1}+r \bar{\lambda} h_{1}, f_{2}+r h_{2}, \ldots, f_{k}+r h_{k}\right)\right\| \\
& \geq\left|T\left(f_{1}+r \bar{\lambda} h_{1}, f_{2}+r h_{2}, \ldots, f_{k}+r h_{k}\right)\left(z_{0}\right)\right| \\
& =\left|T\left(f_{1}, \ldots, f_{k}\right)\left(z_{0}\right)+r^{k} \bar{\lambda} T\left(h_{1}, \ldots, h_{k}\right)\left(z_{0}\right)\right|=\alpha+r^{k},
\end{aligned}
$$

which is a contradiction showing that $T\left(f_{1}, \ldots, f_{k}\right)(z)=0$ for all $z \in \mathcal{I}_{x_{1}, \ldots, x_{k}}$.

Lemma 4.4. Let $\left(x_{1}, \ldots, x_{k}\right)$ and $\left(x_{1}^{\prime}, \ldots, x_{k}^{\prime}\right)$ be distinct points in $C h\left(A_{1}\right) \times \ldots \times C h\left(A_{k}\right)$. Then $\mathcal{I}_{x_{1}, \ldots, x_{k}} \cap \mathcal{I}_{x_{1}^{\prime}, \ldots, x_{k}^{\prime}}=\emptyset$.

Proof. Contrary to what we claim, assume that there exists $z_{0} \in \mathcal{I}_{x_{1}, \ldots, x_{k}} \cap \mathcal{I}_{x_{1}^{\prime}, \ldots, x_{k}^{\prime}}$. Since $\left(x_{1}, \ldots, x_{k}\right)$ and $\left(x_{1}^{\prime}, \ldots, x_{k}^{\prime}\right)$ are distinct, the set $L=\left\{i: 1 \leq i \leq k, x_{i} \neq x_{i}^{\prime}\right\}$ is nonempty. For each $i \in L$, we can choose a function $g_{i} \in A_{i}$ such that $g_{i}\left(x_{i}\right)=1$ and $g_{i}\left(x_{i}^{\prime}\right)=0$, and then, by Lemma 2.1, a peaking function $h_{i} \in P_{A_{i}}\left(x_{i}\right)$ such that $g_{i} h_{i} \in P_{A_{i}}\left(x_{i}\right)$. Now if we let $f_{i}=g_{i} h_{i}$ for every $i \in L$, then $f_{i} \in C_{x_{i}}$ with $f_{i}\left(x_{i}\right)=1$ and $f_{i}\left(x_{i}^{\prime}\right)=0$. Moreover, for each $j \in\{1, \ldots, k\} \backslash L$, we can also choose a peaking function $f_{j} \in C_{x_{j}}$. On one side, since $\left(f_{1}, \ldots, f_{k}\right) \in C_{x_{1}} \times \ldots \times C_{x_{k}}$, $\left|T\left(f_{1}, \ldots, f_{k}\right)\left(z_{0}\right)\right|=1$. On the other side, by Lemma $4.3, T\left(f_{1}, \ldots, f_{k}\right)\left(z_{0}\right)=0$, which is impossible. Therefore, $\mathcal{I}_{x_{1}, \ldots, x_{k}} \cap \mathcal{I}_{x_{1}^{\prime}, \ldots, x_{k}^{\prime}}=\emptyset$.

Theorem 4.5. Suppose that $T: A_{1} \times \ldots \times A_{k} \longrightarrow C_{0}(Z)$ is a k-linear isometry. Then there exist a nonempty subset $Z_{0}$ of $Z$, a continuous surjective $\operatorname{map} \varphi: Z_{0} \longrightarrow C h\left(A_{1}\right) \times \ldots \times C h\left(A_{k}\right)$ and a unimodular continuous function $a: Z_{0} \longrightarrow \mathbb{T}$ such that $T\left(f_{1}, \ldots, f_{k}\right)(z)=a(z) \prod_{i=1}^{k} f_{i}\left(\pi_{i}(\varphi(z))\right)$ for all $\left(f_{1}, \ldots, f_{k}\right) \in A_{1} \times \ldots \times A_{k}$ and $z \in Z_{0}$, where $\pi_{i}$ is the ith projection map.

Proof. Let $Z_{0}:=\left\{z \in \mathcal{I}_{x_{1}, \ldots, x_{k}}:\left(x_{1}, \ldots, x_{k}\right) \in C h\left(A_{1}\right) \times \ldots \times C h\left(A_{k}\right)\right\}$ which is a nonempty set, by Lemma 4.1. Fix $\left(x_{1}, \ldots, x_{k}\right) \in C h\left(A_{1}\right) \times \ldots \times C h\left(A_{k}\right)$ and $h_{i} \in C_{x_{i}}$ with $h_{i}\left(x_{i}\right)=1$ for each $i$, $i=1, \ldots, k$. Then for each $i, i=1, \ldots, k$, we can define an isometry as follows:

$$
\left\{\begin{array}{c}
T_{i}: A_{i} \longrightarrow C_{0}(Z) \\
T_{i}(f)=T\left(h_{1}, \ldots, h_{i-1}, f, h_{i+1}, \ldots, h_{k}\right) . \\
8
\end{array}\right.
$$


According to Theorem 3.1, there exist a subset $Z_{i}$ of $Z$, a continuous surjective map $\varphi_{i}: Z_{i} \longrightarrow$ $C h\left(A_{i}\right)$ such that

$$
T_{i}\left(f_{i}\right)(z)=T\left(h_{1}, \ldots, h_{k}\right)(z) f_{i}\left(\varphi_{i}(z)\right), \quad\left(f_{i} \in A_{i}, z \in Z_{i}\right)
$$

Namely, $Z_{i} \supseteq \bigcup_{x_{i}^{\prime} \in C h\left(A_{i}\right)} \mathcal{I}_{x_{1}, \ldots, x_{i}^{\prime}, \ldots, x_{k}}$ and if $z \in \mathcal{I}_{x_{1}, \ldots, x_{i}^{\prime}, \ldots, x_{k}}$, then $\varphi_{i}(z)=x_{i}^{\prime}$.

Let $\left(f_{1}, \ldots, f_{k}\right) \in A_{1} \times \ldots \times A_{k}$. Now for a given $z \in \mathcal{I}_{x_{1}, \ldots, x_{k}}$, by Lemma 4.3 and using the above reasonings, we conclude that

$$
\begin{aligned}
0 & =T\left(f_{1}-f_{1}\left(x_{1}\right) h_{1}, f_{2}-f_{2}\left(x_{2}\right) h_{2}, h_{3}, \ldots, h_{k}\right)(z) \\
& =T\left(f_{1}, f_{2}, h_{3}, \ldots, h_{k}\right)(z)-f_{1}\left(x_{1}\right) T\left(h_{1}, f_{2}, h_{3}, \ldots, h_{k}\right)(z) \\
& -f_{2}\left(x_{2}\right) T\left(f_{1}, h_{2}, h_{3}, \ldots, h_{k}\right)(z)+f_{1}\left(x_{1}\right) f_{2}\left(x_{2}\right) T\left(h_{1}, \ldots, h_{k}\right)(z) \\
& =T\left(f_{1}, f_{2}, h_{3}, \ldots, h_{k}\right)(z)-f_{1}\left(x_{1}\right) T_{2}\left(f_{2}\right)(z)-f_{2}\left(x_{2}\right) T_{1}\left(f_{1}\right)(z)+f_{1}\left(x_{1}\right) f_{2}\left(x_{2}\right) T\left(h_{1}, \ldots, h_{k}\right)(z) \\
& =T\left(f_{1}, f_{2}, h_{3}, \ldots, h_{k}\right)(z)-f_{1}\left(x_{1}\right) T\left(h_{1}, \ldots, h_{k}\right)(z) f_{2}\left(x_{2}\right) \\
& -f_{2}\left(x_{2}\right) T\left(h_{1}, \ldots, h_{k}\right)(z) f_{1}\left(x_{1}\right)+f_{1}\left(x_{1}\right) f_{2}\left(x_{2}\right) T\left(h_{1}, \ldots, h_{k}\right)(z) \\
& =T\left(f_{1}, f_{2}, h_{3}, \ldots, h_{k}\right)(z)-f_{1}\left(x_{1}\right) f_{2}\left(x_{2}\right) T\left(h_{1}, \ldots, h_{k}\right)(z) .
\end{aligned}
$$

Thus $T\left(f_{1}, f_{2}, h_{3}, \ldots, h_{k}\right)(z)=T\left(h_{1}, \ldots, h_{k}\right)(z) f_{1}\left(x_{1}\right) f_{2}\left(x_{2}\right)$. By continuing this process and applying Lemma 4.3, finally we see that

$$
\begin{aligned}
0 & =T\left(f_{1}-f_{1}\left(x_{1}\right) h_{1}, \ldots, f_{k}-f_{k}\left(x_{k}\right) h_{k}\right)(z) \\
& =T\left(f_{1}, \ldots, f_{k}\right)(z)-T\left(h_{1}, \ldots, h_{k}\right)(z) f_{1}\left(x_{1}\right) \ldots f_{k}\left(x_{k}\right),
\end{aligned}
$$

thereby, $T\left(f_{1}, \ldots, f_{k}\right)(z)=T\left(h_{1}, \ldots, h_{k}\right)(z) f_{1}\left(x_{1}\right) \ldots f_{k}\left(x_{k}\right)$.

Now we define the map $\varphi: Z_{0} \longrightarrow C h\left(A_{1}\right) \times \ldots \times C h\left(A_{k}\right)$ by $\varphi(z):=\left(x_{1}, \ldots, x_{k}\right)$ if $z \in \mathcal{I}_{x_{1}, \ldots, x_{k}}$. Since for distinct points $\left(x_{1}, \ldots, x_{k}\right)$ and $\left(x_{1}^{\prime}, \ldots, x_{k}^{\prime}\right)$ in $C h\left(A_{1}\right) \times \ldots \times C h\left(A_{k}\right)$, Lemma 4.4 yields $\mathcal{I}_{x_{1}, \ldots, x_{k}} \cap \mathcal{I}_{x_{1}^{\prime}, \ldots, x_{k}^{\prime}}=\emptyset$, so the map $\varphi$ is well-defined. Moreover, we can define the unimodular function $a: Z_{0} \longrightarrow \mathbb{T}$ such that if $z \in Z_{0}$ then $a(z):=T\left(h_{1}, \ldots, h_{k}\right)(z)$, where $h_{i} \in P_{A_{i}}\left(\pi_{i}(\varphi(z))\right)$. Lemma 4.3 implies that the definition of $a(z)$ is independent of the choice of $h_{1}, \ldots, h_{k}$. Besides, from the above argument, it follows that if $z \in Z_{0}$ with $\varphi(z)=\left(x_{1}, \ldots, x_{k}\right)$ and $\left(f_{1}, \ldots, f_{k}\right) \in A_{1} \times \ldots \times A_{k}$ then

$$
T\left(f_{1}, \ldots, f_{k}\right)(z)=a(z) \prod_{i=1}^{k} f_{i}\left(x_{i}\right)=a(z) \prod_{i=1}^{k} f_{i}\left(\pi_{i}(\varphi(z))\right) .
$$

Next we prove that $\varphi$ is continuous. Suppose that $z_{0} \in Z_{0}, \varphi\left(z_{0}\right)=\left(x_{1}, \ldots, x_{k}\right)$ and $U_{1} \times \ldots \times U_{k}$ is a neighborhood of $\left(x_{1}, \ldots, x_{k}\right)$ in $C h\left(A_{1}\right) \times \ldots \times C h\left(A_{k}\right)$. For each $i, i=1, \ldots, k$, there is a neighborhood $U_{i}^{\prime}$ of $x_{i}$ in $X_{i}$ with $U_{i}=U_{i}^{\prime} \cap C h\left(A_{i}\right)$. Choose a peaking function $f_{i} \in C_{x_{i}}$ such that $\left|f_{i}\right|<\frac{1}{2}$ on 
$X_{i} \backslash U_{i}^{\prime}(i=1, \ldots, k)$. Then $\left|T\left(f_{1}, \ldots, f_{k}\right)\left(z_{0}\right)\right|=1$. Set

$$
V:=\left\{z \in Z_{0}:\left|T\left(f_{1}, \ldots, f_{k}\right)(z)\right|>\frac{1}{2}\right\} .
$$

Clearly $V$ is a neighborhood of $z_{0}$ such that $\varphi(V) \subseteq U_{1} \times \ldots \times U_{k}$ because if $z \in V$ and $\varphi(z)=$ $\left(x_{1}^{\prime}, \ldots, x_{k}^{\prime}\right)$, then

$$
\frac{1}{2}<\left|T\left(f_{1}, \ldots, f_{k}\right)(z)\right|=\prod_{i=1}^{k}\left|f_{i}\left(x_{i}^{\prime}\right)\right| \leq\left|f_{i}\left(x_{i}^{\prime}\right)\right| \quad(i=1, \ldots, k) .
$$

Hence $x_{i}^{\prime} \in U_{i}$ and so $\left(x_{1}^{\prime}, \ldots, x_{k}^{\prime}\right) \in U_{1} \times \ldots \times U_{k}$.

To complete the proof, it suffices to check the continuity of $a$. Let $z_{0} \in Z_{0}$. Then $z_{0} \in \mathcal{I}_{x_{1}, \ldots, x_{k}}$ for a unique $\left(x_{1}, \ldots, x_{k}\right)$ in $C h\left(A_{1}\right) \times \ldots \times C h\left(A_{k}\right)$. For each $i, i=1, \ldots, k$, choose a peaking function $f_{i} \in P_{A_{i}}\left(x_{i}\right)$ and take

$$
U_{i}:=\left\{x \in C h\left(A_{i}\right): f_{i}(x) \neq 0\right\} .
$$

Then $U=U_{1} \times \ldots \times U_{k}$ is a neighborhood of $\left(x_{1}, \ldots, x_{k}\right)$ in $C h\left(A_{1}\right) \times \ldots \times C h\left(A_{k}\right)$ and consequently $\varphi^{-1}(U)$ is a neighborhood of $z_{0}$. We have

$$
a(z)=\frac{T\left(f_{1}, \ldots, f_{k}\right)(z)}{\prod_{i=1}^{k} f_{i}\left(\pi_{i}(\varphi(z))\right)} \quad\left(z \in \varphi^{-1}(U)\right) .
$$

So from the continuity of the function $\frac{T\left(f_{1}, \ldots, f_{k}\right)}{\prod_{i=1}^{k} f_{i} \circ \pi_{i} \circ \varphi}$ on $\varphi^{-1}(U)$, we conclude that $a$ is continuous at $z_{0}$.

Acknowledgments. We thank the referee for his/her invaluable comments and suggestions.

Research of J.J. Font and M. Sanchis was partially supported by the Spanish Ministry of Science and Education (Grant number MTM2011-23118), and by Bancaixa (Projecte P11B2011-30).

\section{REFERENCES}

[1] J. Araujo and J.J. Font, Linear isometries between subspaces of continuous functions, Trans. Amer. Math. Soc. 349 (1997) 413-428.

[2] J. Araujo and J.J. Font, On S̆ ilov boundaries for subspaces of continuous functions, Topology Appl. 77 (1997) $79-85$.

[3] A. Browder, Introduction to Function Algebras, W. A. Benjamin, New York-Amsterdam, 1969.

[4] H.G. Dales, Boundaries and peak points for Banach function algebras, Proc. London Math. Soc. 22 (1971) 121-136.

[5] R.J. Fleming and J.E. Jamison, Isometries on Banach Spaces: Function Spaces, Chapman Hall/CRC Monogr. Surv. Pure Appl. Math., 129, Chapman Hall/CRC, Boca Raton, 2003.

[6] J.J. Font and M. Sanchis, Bilinear isometries on subspaces of continuous functions, Math. Nachr. 283 (2010) 568-572.

[7] O. Hatori, S. Lambert, A. Lutman, T. Miura, T. Tonev, and R.Yates, Spectral preservers in commutative Banach algebras, Contemp. Math. 547 (2011) 103-123. 
[8] O. Hatori, T. Miura, and H. Takagi, Characterizations of isometric isomorphisms between uniform algebras via nonlinear range-preserving properties, Proc. Amer. Math. Soc. 134 (2006) 2923-2930.

[9] W. Holsztyński, Continuous mappings induced by isometries of spaces of continuous functions, Studia Math. 26 (1966) 133-136.

[10] A. Moreno and A. Rodríguez, A bilinear version of Holsztyński's theorem on isometries of $C(X)$-spaces, Studia Math. 166 (2005) 83-91.

[11] N.V. Rao and A.K. Roy, Multiplicatively spectrum-preserving maps of function algebras II, Proc. Edinb. Math. Soc. 48 (2005) 219-229.

[12] T. Tonev and R. Yates, Norm-linear and norm-additive operators between uniform algebras, J. Math. Anal. Appl. 357 (2009) 45-53.

Department of Mathematics, K.N. Toosi University of Technology, Tehran, 16315-1618, Iran

E-mail address: m.hosseini@kntu.ac.ir

Departamento de Matemáticas, Universitat Jaume I, Campus Riu Sec, 8029 AP, Castellón, Spain

E-mail address: font@mat.uji.es

Departamento de Matemáticas, Universitat Jaume i, Campus Riu Sec, 8029 AP, Castellón, Spain

E-mail address: sanchis@mat.uji.es 